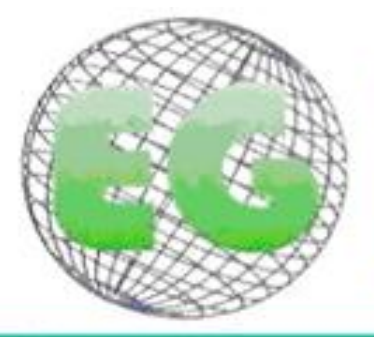

$N^{0} 32$

\title{
Lesiones osteomusculares de miembros superiores y región lumbar: caracterización demográfica y ocupacional. Universidad Nacional de Colombia, Bogotá 2001- 2009
}

Musculoskeletal disorders of the upper and the lumbar region: demographic and occupational characterization, Universidad Nacional de Colombia, Bogotá 2001- 2009

\section{*Vargas Porras, PA ** Orjuela Ramírez, ME *** Vargas Porras, C \\ *Fisioterapeuta. Magíster en Salud y Seguridad en el Trabajo. **Doctor em Salud Pública.Enfermera. Profesor Universidad Nacional de Colombia, Bogotá *** Magíster en Enfermería. Profesora Escuela de Enfermería, Facultad de Salud, Universidad Industrial de Santander, Bucaramanga. Colombia. E-mail:} cvargasporras@yahoo.com

Palabras clave: lesiones osteomusculares; miembros superiores; región lumbar; factor de riesgo; ocupacional.

Keywords: Musculoskeletal injuries; upper limbs; lumbar region; risk factor; occupational.

\section{RESUMEN}

Objetivo: Caracterizar variables demográficas y ocupacionales de casos de lesiones osteomusculares de miembros superiores y región lumbar.

Metodología: Estudio descriptivo, corte transversal. Se realizó búsqueda y revisión de literatura científica para conocer factores de riesgo laborales asociados a lesiones osteomusculares de miembros superiores, región lumbar y determinar variables relevantes. Las variables demográficas y ocupacionales en casos de lesiones osteomusculares, se obtuvieron de registros de la base de datos diseñada por la División Nacional de Salud Ocupacional de la Universidad Nacional de Colombia.

Resultados: Se identificaron 99 registros de trabajadores con diagnóstico positivo de lesiones osteomusculares de miembros superiores y región lumbar. El género femenino y grupo etáreo entre 41 a 50 años reportó $60 \%$ y $43.43 \%$ de casos de lesiones osteomusculares respectivamente. El porcentaje de casos según grupo ocupacional para docentes y otros administrativos presentó distribución similar $28.28 \%$. Se encontró que movimientos repetitivos de codo, muñeca, dedos para miembros superiores y postura prolongada para región lumbar son factores de riesgo más frecuentes. En trabajadores los segmentos corporales más comprometidos con lesiones osteomusculares fueron muñeca y zona lumbar. Escoliosis e hiperlordosis fueron las alteraciones de columna vertebral con mayor frecuencia entre la población participante. La mejoría y/o desaparición de los síntomas con descanso y reaparición o agravamiento durante el trabajo fue el criterio más representativo para considerar posible origen laboral de casos de lesiones osteomusculares para miembros superiores y región lumbar. 
Conclusión: El conocimiento de las variables asociadas a lesiones osteomusculares permite realizar intervenciones preventivas.

\title{
ABSTRACT
}

The aim of this study was to characterize demographic and occupational variables of cases of upper limb and lower back musculoskeletal injuries.

\begin{abstract}
Methodology: In a descriptive, cross-sectional study, we searched and reviewed scientific literature to learn about occupational risk factors associated with musculoskeletal injuries of upper limbs, the lower back and to determine relevant variables. The demographic and occupational variables in musculoskeletal injury cases were obtained from records in the database designed by the National Occupational Health Division at Universidad Nacional de Colombia.
\end{abstract}

Results: We identified 99 records of workers with positive diagnosis of upper limb and lower back musculoskeletal injuries. The female gender and age group between 41 to 50 years reported $60 \%$ and $43.43 \%$ of the cases of musculoskeletal injuries respectively. The percentage of cases by occupational group for teachers and administrative workers showed a similar distribution of $28.28 \%$. We found that repetitive movements of elbow, wrist, fingers for upper limbs and an extended position for lumbar region are the most common risk factors. In workers, wrist and lower back were the most prominent musculoskeletal injuries. Scoliosis and hyperlordosis were spinal disorders more frequent among participants. The improvement and / or disappearance of symptoms with rest and recurrence or worsening during the work time was the more representative criterion for considering the possible cases of occupational origin of musculoskeletal injuries to upper limbs and lower back.

Conclusion: The knowledge of these variables associated with musculoskeletal injuries allows for preventive interventions.

\section{INTRODUCCIÓN}

La Organización Internacional del Trabajo (OIT), según el informe publicado durante el año 2005 expone que cada día muere un promedio de 5.000 personas como resultado de accidentes o dolencias relacionadas con el trabajo. La OIT estima que esta cifra es de 2 a 2,3 millones de hombres y mujeres al año, de los cuales 350.000 corresponden a muertes por accidentes en el trabajo y de 1,7 a 2 millones corresponden a muertes ocasionadas por enfermedades contraídas por el tipo de trabajo; este mismo informe precisa que, adicionalmente, los trabajadores del mundo sufren 270 millones de accidentes ocupacionales, y se producen cerca de 160 millones de casos de enfermedades con consecuencias no fatales. ${ }^{(1)}$

En relación con las enfermedades del sistema músculo-esquelético, la Conferencia Internacional del Trabajo realizada en 2002, precisó que son aquellas enfermedades causadas por ciertas actividades laborales o por factores de riesgo presentes en el ambiente de trabajo. (2)

Al respecto es conveniente mencionar que en el informe de enfermedad profesional en Colombia 2003-2005, ${ }^{(3)}$ el síndrome del conducto carpiano (SCC) se constituye en la primera causa de morbilidad profesional. Durante el año 2004 el 32\% de los diagnósticos de enfermedad profesional correspondió a la mencionada patología, observándose un incremento de manera constante.

Por otra parte, el lumbago que se incluye dentro de las lesiones osteomusculares y ligamentosas de la tabla de enfermedades ocupacionales, continúa ocupando el segundo lugar en frecuencia de diagnóstico de enfermedades profesionales. Así, por ejemplo, durante el año 2004, el 15\% de los diagnósticos correspondió a esta 
patología, sin embargo, es de resaltar que el diagnóstico de lumbago es un diagnóstico inespecífico y que tal vez por tal razón su incidencia disminuye en el año 2004 con respecto a los tres años anteriores. En este contexto, al agrupar los diagnósticos por sistemas, se observa que el sistema músculo esquelético es el más afectado y según el reporte de accidentes de trabajo y enfermedades profesionales del Ministerio de Protección Social, se observa el incremento constante de los desórdenes musculoesqueléticos de origen ocupacional (DME), los cuales pasaron del $65 \%$ en el año 2001 , al $82 \%$ de todos los diagnósticos en el año 2004. Estos DME afectan básicamente dos segmentos corporales: miembro superior y columna lumbosacra. ${ }^{(4)}$

Cabe señalar que entre las múltiples repercusiones ocasionadas por las lesiones músculo esqueléticas entre los trabajadores, debido a los factores de riesgo ocupacionales, se diferencian fundamentalmente: la modificación de la calidad de vida del trabajador, los cambios en las perspectivas y actitudes psicosociales individuales, familiares y sociales, el ausentismo, la disminución de la productividad, y el aumento de los costos económicos, de los cuidados a la salud.

La Universidad Nacional de Colombia como institución del sector público y obedeciendo las normas legales vigentes en materia de salud ocupacional, ha definido como política de salud ocupacional la protección integral de la salud, la prevención y control de riesgos profesionales del personal docente, administrativo, contratistas y terceros. Así mismo, la División Nacional de Salud Ocupacional es una unidad adscrita a la División Nacional de Personal, que tiene como objetivo orientar sus acciones hacia la prevención y control de factores de riesgo ocupacionales, favoreciendo un ambiente laboral seguro, que facilite y proteja la salud integral de los servidores públicos docentes y administrativos. ${ }^{(5)}$

Teniendo en cuenta que la protección integral de la salud es una política de salud ocupacional definida por la Universidad Nacional de Colombia, en este contexto, se diseñó e implementó el Sistema de Vigilancia Ocupacional de Trastornos y Lesiones Osteomusculares (SIVIGOTOM).

EI SIVIGOTOM, tuvo como población objeto de vigilancia a todos los trabajadores de la Universidad Nacional de Colombia -sede Bogotá-, incluyó por lo tanto a los trabajadores administrativos y docentes que laboran en las instalaciones dentro y fuera del campus universitario, la finalidad del sistema fue describir la ocurrencia de los trastornos osteomusculares entre los trabajadores y sugerir los lineamientos que tengan por objetivo prevenir la aparición de dichas lesiones. La decisión de optar por este tipo de alteraciones de la salud se basó en un diagnóstico previo de las condiciones de salud y trabajo de tres grupos considerados como de alto riesgo para la ocurrencia de alteraciones ostemusculares, que correspondió a los trabajadores que cumplen labores de mantenimiento, servicios generales y secretariales. ${ }^{(6)}$

Cabe señalar que el dolor lumbar es la principal causa de ausentismo por enfermedad y discapacidad en las poblaciones trabajadoras. Se considera que aproximadamente entre el 10 y el 15\% de la población general en algún momento de la vida manifiesta haber tenido un dolor lumbar; entre los trabajadores se considera que cerca del $2 \%$ tienen que ausentarse del trabajo por causa del dolor lumbar cada año. Por otra parte los síndromes dolorosos del miembro superior configuran el grupo de lesiones osteomusculares más frecuentes. La fisiopatología de estos síndromes en la actualidad no es comprendida en su totalidad; se consideran cinco factores de riesgo 
ocupacionales importantes en el desarrollo de este tipo de entidades: la repetición, la fuerza, la postura, el estrés, y la vibración. ${ }^{(6)}$

A partir de la aplicación de las pruebas de tamizaje y evaluaciones fisiocinéticas para miembros superiores y región lumbar a un total de 2.117 trabajadores de la Universidad Nacional de Colombia -sede Bogotá- desde el 2001 al 2009, se identificó que la prevalencia de dolor lumbar fue del $18.79 \%$, y de un $61.40 \%$ con relación a síndromes dolorosos de miembros superiores. ${ }^{(6)}$ Sin embargo, no se tiene información de los resultados de informes que posteriormente hayan reportado cambios o modificaciones en esta tendencia.

Las lesiones osteomusculares han sido definidas por el instituto Nacional para la Seguridad y Salud Ocupacional $(\mathrm{NIOSH})^{(7)}$ como "un grupo de condiciones que involucra a los nervios, tendones, músculos y estructuras de soporte como los discos intervertebrales". A su vez, la Organización Mundial de la Salud (OMS) precisa que las lesiones osteomusculares hacen parte de un grupo de condiciones relacionadass con el trabajo, porque ellas pueden ser causadas tanto por exposiciones ocupacionales como por exposiciones no ocupacionales." (8)

Es evidente que las lesiones osteomusculares son un problema de salud pública que se destacan e imponen una enorme carga para los trabajadores y para la sociedad, afectan la calidad de vida, reducen la productividad y rentabilidad, producen incapacidad temporal o permanente, inhabilitan para la realización de tareas e incrementan los costos de compensación al trabajador.

En este marco de referencia, y dado que se carece de información sobre las características demográficas y ocupacionales de los casos identificados de lesiones osteomusculares de miembros superiores y región lumbar en este grupo laboral, es necesario identificar la población laboral de acuerdo con el riesgo de exposición, e identificar factores de riesgo asociados con los casos reconocidos en las pruebas tamiz.

La caracterización de las variables demográficas y ocupacionales de los casos de lesiones osteomusculares y los factores de riesgo ocupacionales, constituye un principio fundamental para el análisis de las condiciones de trabajo y salud en actividades económicas específicas, puesto que con base en esta información, se definen las medidas de intervención concretas que contribuyen a solucionar las implicaciones de las alteraciones osteomusculares, influyendo significativamente en la disminución de las cifras de morbilidad laboral.

En este sentido, la caracterización demográfica y ocupacional de los casos de lesiones osteomusculares, proporcionará información objetiva y específica de los grupos ocupacionales vulnerables, contribuyendo así en la planeación de las actividades de prevención, control e intervención acorde a las necesidades propias de este grupo laboral, apoyando en el mejoramiento de las condiciones de trabajo y salud y por ende la calidad de vida y bienestar de dicha población.

El presente trabajo tuvo como objetivo caracterizar las variables demográficas y ocupacionales de los casos de lesiones osteomusculares de miembros superiores y región lumbar, incluidos en el Sistema de Vigilancia Ocupacional de Trastornos y Lesiones Osteomusculares (SIVIGOTOM), en el periodo comprendido entre el año 2001 al 2009 y con ello describir las variables significativas de persona, tiempo y lugar 
en los casos identificados por las valoraciones fisiocinéticas en la población objeto de estudio, y señalar los factores de riesgo laboral.

\section{MATERIALES Y MÉTODOS}

Se realizó un estudio descriptivo de corte transversal, dirigido a la caracterización demográfica y ocupacional de los casos de lesiones osteomusculares identificados mediante la aplicación de pruebas tamiz a través de la valoración fisiocinéticas a funcionarios del área administrativa, que laboran en la Universidad Nacional de Colombia y que fueron incluidos dentro de Sistema de Vigilancia Ocupacional de Trastornos y Lesiones Osteomusculares (SIVIGOTOM), en el periodo comprendido entre el año 2001 al 2009.

Las variables demográficas y ocupacionales en los casos de lesiones osteomusculares de miembros superiores y región lumbar en los trabajadores de la Universidad Nacional de Colombia -sede Bogotá-, provienen de los registros de la base de datos diseñada por la División Nacional de Salud Ocupacional de la Universidad Nacional de Colombia para el Sistema de Vigilancia Ocupacional de Trastornos y Lesiones Osteomusculares (SIVIGOTOM).

Se realizó la revisión de 2669 registros en la base de datos de los trabajadores incluidos en el sistema de vigilancia ocupacional de trastornos y lesiones osteomusculares. Se excluyeron 552 registros con información incompleta o duplicada. En los restantes 2117 registros, se identificaron 99 registros de trabajadores a quienes mediante la prueba tamiz de la valoración fisiocinética presentaron un diagnóstico positivo de lesiones osteomusculares de miembros superiores y región lumbar.

Para el análisis de los resultados se usó la distribución de frecuencias y porcentajes de los casos de lesiones osteomusculares de miembros superiores y región lumbar según grupo etáreo, género, grupo ocupacional, antigüedad laboral en la Universidad y exposición a factores de riesgo laboral en miembros superiores y región lumbar.

Se realizó una búsqueda y revisión de la literatura científica en la base de datos Pub Med con el objetivo de conocer los factores de riesgo laborales asociados a lesiones osteomusculares de miembros superiores y región lumbar, y determinar las variables relevantes para el estudio.

Para la búsqueda de los artículos desde el año 2005 hasta la fecha, se utilizaron los siguientes parámetros: Full text, Humanos. Type of article: Clinical trial, Meta-analysis, Randomized controlled trial, Review, Journal Article. Descriptores: incidence, disorder, musculoskeletal, occupational, lumbar spine, upper limbs, secretaries, teacher, administrative staff, personal hygiene.

Los resultados mostraron que 150.209 artículos publicados relacionados (lesiones osteomusculares, lesiones osteomusculares ocupacionales, incidencia de lesiones osteomusculares, incidencia de lesiones osteomusculares ocupacionales) de estos $98.64 \%$ correspondió a lesiones osteomusculares y sólo $0.60 \%$ a la incidencia de estas lesiones.

De la misma manera $7.16 \%$ de los artículos de lesiones osteomusculares hizo referencia a lesiones de columna lumbar y $4.26 \%$ a miembros superiores. Un $1.8 \%$ y 
$32.88 \%$ de los artículos concernientes a lesiones osteomusculares ocupacionales correspondieron a columna lumbar y miembros superiores, respectivamente. De 914 artículos propios a incidencia de lesiones osteomusculares, $3.06 \%$ correspondió a la columna lumbar, $13.78 \%$ a miembros superiores, finalmente el $42.56 \%$ se relacionó a incidencia ocupacional.

Por otra parte, $33 \%$ estudiaron la relación de los trastornos musculoesqueléticos con género, $42 \%$ los grupos ocupacionales, $20 \%$ los factores físicos, $25 \%$ las condiciones ergonómicas y es de resaltar que los factores psicosociales correspondieron al $66 \%$ de los artículos hallados.

Se elaboró la correspondiente base de datos en Excel mediante la utilización de códigos.

\section{Aspectos éticos}

Se siguieron los lineamientos de la Resolución $008430,{ }^{(9)}$ en la cual se establecen las normas científicas, técnicas y administrativas para la investigación en salud. Según esta resolución se clasifica como una investigación sin riesgo. Se tuvieron en cuenta las pautas éticas internacionales para la investigación biomédica en seres humanos recomendadas por CIOMS. ${ }^{(10)}$ Se utilizaron los datos generales de los registros, lo cual garantizó la confidencialidad de la información dado que no se individualizaron los casos de lesiones osteomusculares. Contó con el aval del comité de ética de la Facultad de Enfermería de la Universidad Nacional de Colombia.

\section{RESULTADOS}

En la distribución porcentual según grupo etáreo de los casos de lesiones osteomusculares de miembros superiores y región lumbar en trabajadores se observó que $43.43 \%$ de los casos de lesiones osteomusculares se diagnosticaron entre el rango de edad de 41 a 50 años, en un segundo lugar los casos se presentaron entre el rango de edades de 31 a 40 años y 51 a 60 años con el $26.26 \%$ respectivamente. El mayor número de casos se identificó en mujeres.

Se presenta en similar proporción el número de casos en docentes y otros administrativos, seguidos por los del grupo de laboratoristas y directivos respectivamente.

La distribución porcentual de los casos de lesiones osteomusculares según exposición laboral antes de ingresar a la Universidad Nacional de Colombia, evidenció que $28,28 \%$ corresponde a un periodo entre 1 a 5 años, seguido de 6 a 10 años con una representación de $20,20 \%$. En la exposición laboral dentro de la Universidad se aprecia que la proporción más alta de los trabajadores se encuentran vinculados desde hace más de 16 años. Seguidos en orden de frecuencia de quienes tienen entre 11 a 15 años. Es decir, que más del $60 \%$ de los casos diagnosticados tienen un tiempo de vinculación de más de 11 años. 


\section{Figura 1. Frecuencia de casos de lesiones osteomusculares de miembros superiores según exposición a factores de riesgo laboral}

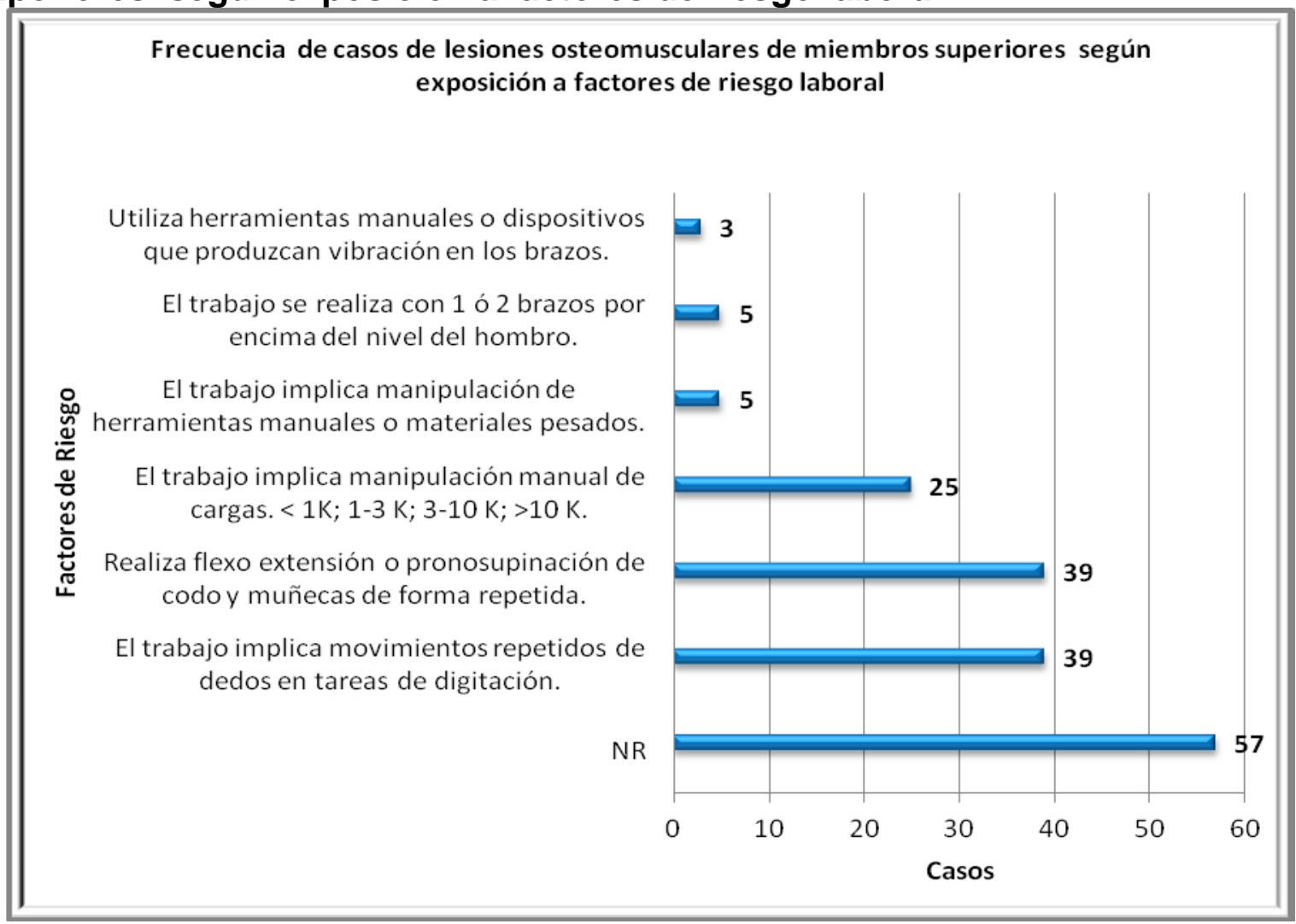

$\mathrm{N}=173^{*}$

*Los trabajadores podían estar expuestos a más de un factor de riesgo laboral

Fuente: registros del Sistema de Vigilancia de Trastornos y Lesiones Osteomusculares (SIVIGOTOM) de la Universidad Nacional de Colombia- sede Bogotá.

Los casos de lesiones osteomusculares de miembros superiores según exposición a factores de riesgo laboral, se presentan en la figura 1. Se observa que los movimientos repetitivos de codo, muñeca y dedos es el factor de riesgo que presenta mayor frecuencia, seguido de la manipulación manual de cargas, en menor proporción de la manipulación de herramientas manuales y de la ejecución de tareas por encima del nivel del hombro. 
Figura 2. Frecuencia de casos de lesiones osteomusculares de región lumbar según exposición a factores de riesgo laboral

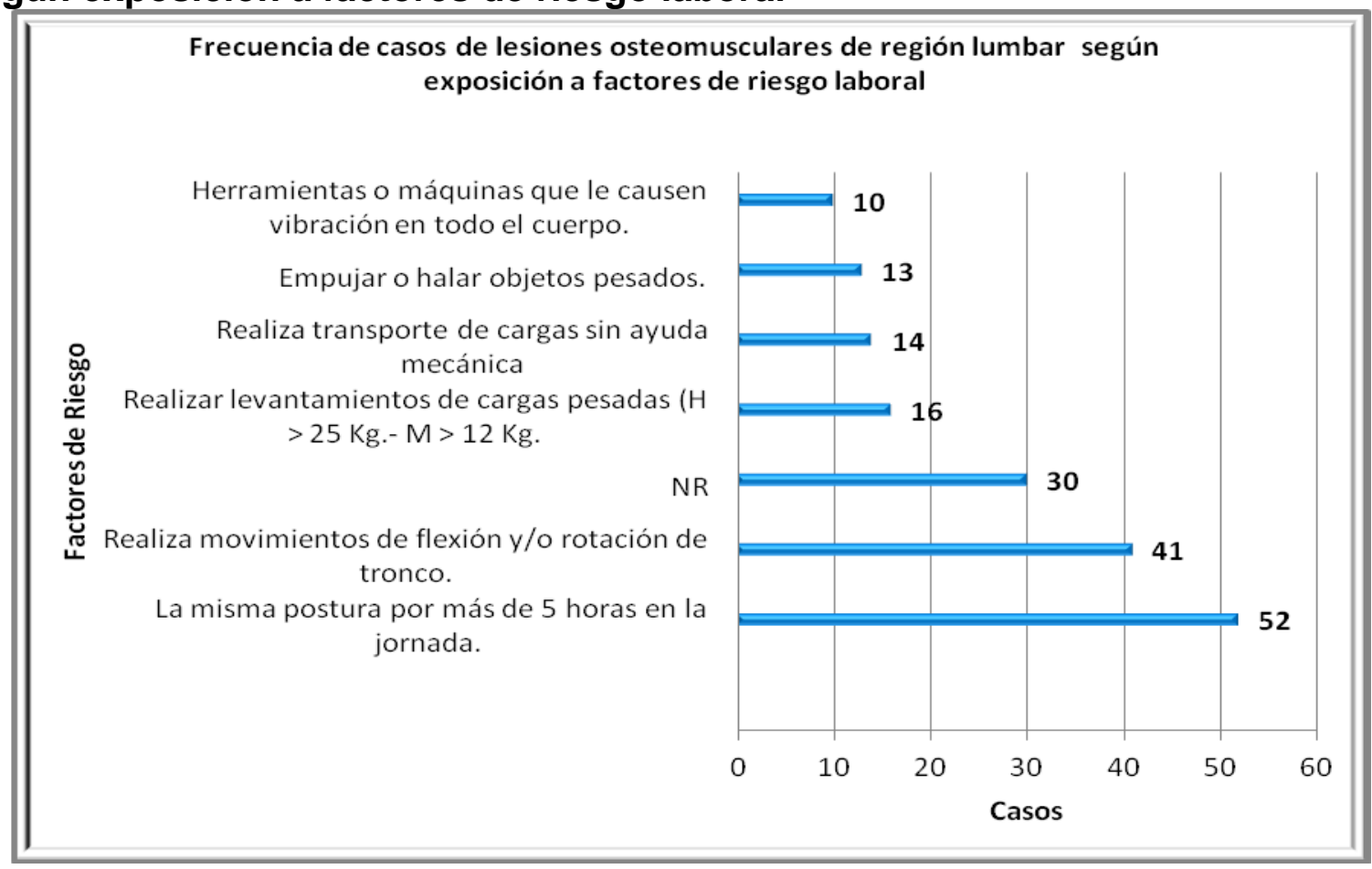

\section{$N=176^{*}$}

* Los trabajadores podían estar expuestos a más de un factor de riesgo laboral

Fuente: registros del Sistema de Vigilancia de Trastornos y Lesiones Osteomusculares (SIVIGOTOM) de la Universidad Nacional de Colombia- sede Bogotá.

La frecuencia de casos de lesiones osteomusculares de región lumbar según exposición a factores de riesgo laboral en la figura 2, muestra que mantener la misma postura por más de 5 horas en la jornada es el factor de riesgo laboral más frecuente al cual están expuestos los trabajadores de la Universidad Nacional de Colombia, seguido de los movimientos de flexión y/o rotación de tronco, en menor proporción se encontró el levantamiento y transporte manual de cargas.

En la distribución porcentual según práctica de actividad física de los casos de lesiones osteomusculares, se aprecia que $53,54 \%$ de los casos realizaban algún tipo de actividad física, comparado con un $46.46 \%$ que no realizaban algún tipo de actividad física.

En los casos de lesiones osteomusculares de miembros superiores y región lumbar según localización del dolor, se observa que los segmentos corporales más comprometidos son la zona lumbar, seguido de la muñeca y el hombro. En relación a la intensidad del dolor, se evidencia que el dato de mayor proporción es el no registro; sin embargo, la intensidad de dolor se ubica en un nivel medio para gran parte de los casos.

En la frecuencia de casos de lesiones osteomusculares de miembros superiores y región lumbar según alteración postural, se destaca que la hiperlordosis y escoliosis son las alteraciones posturales más frecuentes. 
Figura 3. Frecuencia de casos de lesiones osteomusculares de miembros superiores y región lumbar según criterios para considerar posible origen laboral

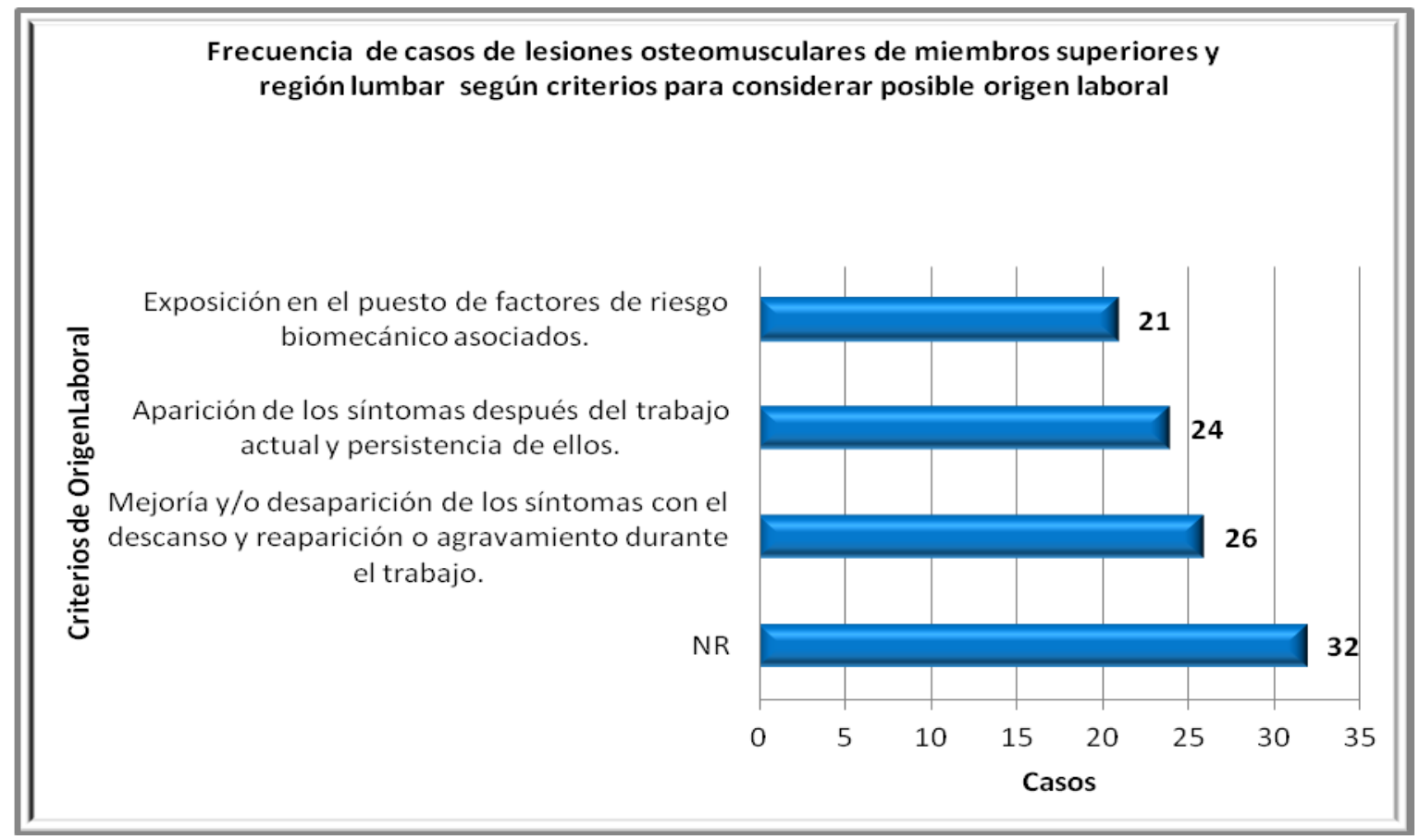

$\mathrm{N}=103^{*}$

*Los trabajadores podían ofrecer más de una respuesta

Fuente: registros del Sistema de Vigilancia de Trastornos y Lesiones Osteomusculares (SIVIGOTOM) de la Universidad Nacional de Colombia- sede Bogotá.

En relación a los criterios para considerar posible origen laboral de los casos de lesiones osteomusculares para miembros superiores y región lumbar, presentados en la figura 3, se puede observar que el no registro de esta información es el porcentaje más alto, de igual forma la mejoría y/o desaparición de los síntomas con el descanso y reaparición o agravamiento durante el trabajo se presentó en 26 de los casos.

Al comparar la distribución porcentual de los casos de lesiones osteomusculares de acuerdo con el género según grupo etáreo, se observa que se presenta un mayor número de casos de lesiones osteomusculares en mujeres y hombres entre las edades de 41 a 50 años de edad; de igual forma se presenta igual número de casos de lesiones osteomusculares tanto en mujeres como en hombres entre las edades de 31 a 40 años y 51 a 60 años.

En la comparación de la distribución porcentual de los casos de lesiones osteomusculares de acuerdo con el género según grupo ocupacional, se presentan un mayor número de casos (25\%) en las mujeres pertenecientes al grupo ocupacional de laboratorista y en igual proporción (25\%) en los hombres pertenecientes al grupo de docentes.

En la comparación de la frecuencia de casos de lesiones osteomusculares de acuerdo con el género según exposición a factores de riesgo laboral para miembros superiores, se evidencia que los movimientos repetitivos de codos, muñecas y dedos es el factor de riesgo al que más están expuestos tanto mujeres como hombres. 
En la comparación de la frecuencia de casos de lesiones osteomusculares de acuerdo con el género según exposición a factores de riesgo laboral para región lumbar, se puede observar que las mujeres están más expuestas a el factor de riesgo de mantenimiento de la misma postura por más de 5 horas en la jornada laboral, y los hombres están más expuestos al factor de riesgo de movimientos de flexión y/o rotación de tronco.

En la comparación de la frecuencia de casos de lesiones osteomusculares de acuerdo al grupo ocupacional según exposición a factores de riesgo laboral para miembros superiores, se evidencia que los casos pertenecientes al grupo otros administrativos, directivos y docentes son los mayores expuestos a factores de riesgo como: movimientos repetitivos de dedos, flexo-extensión o prono-supinación de codo y muñeca de forma repetida y manipulación manual de cargas.

Y finalmente, en la comparación de la frecuencia de casos de lesiones osteomusculares de acuerdo al grupo ocupacional según exposición a factores de riesgo laboral para región lumbar, se observa que los casos pertenecientes al grupo otros administrativos y docentes, están expuestos a movimientos de flexión y/o rotación de tronco, seguido del mantenimiento de la misma postura por más de 5 horas en la jornada laboral; de igual forma, se aprecia que el grupo de laboratorista, está expuesto a la mayoría de los factores de riesgo para lesiones osteomusculares de región lumbar, como lo son levantamiento de cargas pesadas, movimientos de flexión y/o rotación de tronco, transporte de carga sin ayuda mecánica y mantenimiento de la misma postura por más de 5 horas en la jornada laboral.

\section{DISCUSIÓN}

Los resultados de esta investigación muestran la presencia de lesiones osteomusculares especialmente en mujeres y hombres con edades entre 41 a 50 años, este hallazgo concuerda con lo señalado por la Federación de Aseguradores Colombianos (FASECOLDA) que para el año 2007, la proporción de enfermedades profesionales por género fue del $55.2 \%$ para mujeres con una mayor frecuencia de casos entre las edades de 35 a 44 años y del $44.8 \%$ para hombres en este mismo rango de edad. ${ }^{(11)}$

Cabe señalar que el género femenino resulta ser el más afectado con la presencia de lesiones osteomusculares; en este sentido es importante precisar que existen diferencias en la exposición a factores de riesgo según el género; los hombres tienen trabajos que suelen requerir mayor esfuerzo físico, y están expuestos a un mayor número de factores (químicos, físicos, accidentes, etc.); por otra parte, los factores de riesgo más frecuentes en las mujeres trabajadoras son de tipo psicosocial: el alto nivel de exigencia, la monotonía, el sedentarismo, las posturas forzadas, la necesidad de rapidez y destreza en el puesto de trabajo, la poca cualificación y responsabilidad, la acumulación de tareas, y la inseguridad de mantenimiento del puesto. ${ }^{(2,13)}$

En Colombia, según el Ministerio de Protección Social en el informe de enfermedad profesional 2003 - 2005, la prevalencia de enfermedad profesional en mujeres fue de $56 \%$, mientras que en hombres fue de $37 \%$. Es decir, que a diferencia de los accidentes de trabajo las enfermedades profesionales afectan más a las mujeres trabajadoras, a expensas de los desórdenes musculoesqueléticos. ${ }^{(3)}$ Varios estudios han concluido que las lesiones osteomusculares son más comunes entre las mujeres 
que entre los hombres, y que la brecha de género en las lesiones osteomusculares se ha solidificado durante la última década. ${ }^{(14)}$

Hay diversas explicaciones para las diferencias de género en las lesiones osteomusculares, entre ellas se encuentran la segregación de género en el mercado laboral, condiciones de calidad en el trabajo; de igual forma existe una división sexual del trabajo en la esfera de la vida privada resultando en una "doble carga", así como reducidas posibilidades de descanso y recuperación en la mujer después del trabajo. ${ }^{(15,16,17,18)}$

Así mismo, se observó que los grupos ocupacionales con mayor número de casos de lesiones osteomusculares para miembros superiores y región lumbar correspondió a docentes y otros administrativos; el instituto Nacional para la Seguridad y Salud Ocupacional (NIOSH) ${ }^{(7)}$ refiere que los factores de riesgo para desórdenes osteomusculares se relacionan con los esfuerzos prolongados, los movimientos repetitivos, las posiciones incómodas prolongadas y la vibración. Los trabajos o condiciones de trabajos que combinen factores de riesgo aumentarán el riesgo de lesiones osteomusculares, el nivel de riesgo dependerá de cuánto tiempo el trabajador está expuesto a estas condiciones, cuán a menudo está expuesto, y el nivel de exposición.

Sin embargo, Leijon, et al. ${ }^{(15)}$ afirman que "ninguno de los más comunes desórdenes osteomusculares es únicamente explicado por los factores de riesgo en el trabajo". En general, se consideran cuatro grandes grupos de riesgo: los factores ligados a las condiciones de trabajo (carga física: conjunto de requerimientos físicos a los que está sometido el trabajador durante la jornada laboral, se basa en el trabajo muscular estático y dinámico), los factores organizacionales y psicolaborales, los factores relacionados con las condiciones ambientales de los puestos y los sistemas de trabajo (temperatura, vibración, entre otros) y los factores individuales (capacidad funcional del trabajador, hábitos, antecedentes, aspectos psicológicos, etc.).

En este sentido, se pudo apreciar que en los trabajadores pertenecientes al grupo ocupacional de docentes y otros administrativos puede existir una relación en la repetitividad y monotonía de las tareas, posturas prolongadas, estrés y sobrecarga laboral. ${ }^{(18,19,20)}$ La relación entre las lesiones osteomusculares y la ocupación se ha investigado en numerosos estudios, tradicionalmente estos desórdenes se han relacionado con factores físicos del entorno laboral; sin embargo, durante los últimos años, día a día, dicha relación entre los factores psicosociales y las lesiones osteomusculares ha llamado cada vez más la atención ${ }^{(19)}$, las altas demandas psicológicas, el estrés, la percepción del estado de salud, la sobrecarga laboral, las relaciones interpersonales, la pérdida del control sobre las tareas, las largas horas laboradas, la introducción de la tecnología en la producción, el clima organizacional, la pérdida del apoyo social y muchas restricciones situacionales del ambiente de trabajo se involucran como factores psicosociales potenciales para el desarrollo de lesiones osteomusculares. De igual forma aquellos aspectos relacionados con la esfera de la vida privada, tales como, las responsabilidades domésticas y familiares, el tiempo restringido para la recreación y el descanso, están asociadas con un mayor riesgo de lesiones osteomusculares en miembros superiores y columna lumbar ${ }^{(15,16,17}$, $19,20,21,22,23)$.

En este estudio se identificó que los factores de riesgo ocupacionales relacionados con el desarrollo de lesiones osteomusculares fue la exposición de los trabajadores a 
movimientos repetitivos, transporte y manipulación de cargas y posturas prolongadas, tanto para miembros superiores como para la región lumbar, aumentando de esta manera la posibilidad de desarrollar lesiones osteomusculares, a este respecto, diversas publicaciones internacionales señalan como factores de riesgo laborales, asociados al desarrollo de lesiones musculoesqueléticos por esfuerzo físico las tareas que involucran: movimientos repetitivos, posiciones antigravitatorias y prolongadas, levantamiento, transporte o desplazamiento de cargas y exposición a la vibración, los cuales pueden afectar a la mitad de los trabajadores de alto riesgo. ${ }^{(14,24,25,26,27)}$ La incidencia de lesiones osteomusculares en miembros superiores está fuertemente asociada con la exposición a factores de estrés ergonómico como lo son: movimientos repetitivos, posturas corporales forzadas 0 esfuerzos intensos, presentes especialmente en trabajos de oficinas. ${ }^{(17,18,20)}$

A nivel nacional, según la primera encuesta nacional de condiciones de salud y trabajo en el sistema general de riesgos profesionales (SGRP), se encontró que los factores de riesgo ocupacionales más frecuentes fueron aquellos relacionados con las condiciones ergonómicas (carga física), seguidos por los factores de riesgo psicosocial. $^{(28)}$

Por último, se pudo observar que al igual que en el reporte de accidentes de trabajo y enfermedades profesionales del Ministerio de Protección Social del año 2006, en los trabajadores de la Universidad Nacional de Colombia sede Bogotá, los segmentos corporales más comprometidos con respecto a lesiones osteomusculares fueron: la muñeca y la zona lumbar. En España, Torada y Moreno ${ }^{(29)}$ resaltan que las dolencias generadas por las lesiones osteomusculares se presentan en diferentes zonas corporales en hombres y mujeres, mientras que los primeros se ven más afectados en la zona lumbar, las mujeres localizan más molestias en el cuello, zona dorsal, lumbar y miembros superiores.

\section{CONCLUSIONES}

El factor de riesgo con mayor relevancia en los casos de lesiones osteomusculares para miembros superiores sin distinción para ambos géneros es el movimiento repetitivo en codo, muñeca y dedos, presente en los grupos ocupacionales como: otros administrativos, directivos y docentes. El factor de riesgo con mayor relevancia en los casos de lesiones osteomusculares para la región lumbar es la exposición a posturas prolongadas en el caso del género femenino y movimientos de flexión y/o rotación de tronco en el masculino identificándose en docentes, laboratoristas y otros administrativos. El conocimiento de los factores asociados a lesiones osteomusculares de miembros superiores y región lumbar, permite al personal de salud realizar intervenciones preventivas en los trabajadores, teniendo en cuenta tanto en los puestos de trabajo, como las condiciones laborales.

\section{BILIOGRAFÍA}

1.Organización Internacional del Trabajo. Informe publicado con ocasión del "Día Mundial de la Seguridad y la Salud en el Trabajo, 2005". Ginebra. 2005: 107-108. 2.Montoya MC, Palucci H, Cruz ML, Taubert FC. Lesiones osteomusculares en trabajadores de un hospital mexicano y la ocurrencia del ausentismo. Cienc. enferm. 2010; XVI (2): 35-46 [citado en 13 de enero de 2011], [en línea] disponible en: http://www.scielo.cl/pdf/cienf/v16n2/art 05.pdf 
3. Ministerio de la Protección Social. Dirección General de Riesgos Profesionales. "Informe de Enfermedad Profesional en Colombia 2003-2005". Imprenta Nacional de Colombia. Bogotá. 2007; 22 - 25.

4.Ministerio de la Protección Social. Dirección General de Riesgos Profesionales. "Reporte de accidente de trabajo y enfermedad profesional, una oportunidad para la prevención." Bogotá. 2006; 2 - 3.

5.Universidad Nacional de Colombia. [Citado en 15 de enero de 2011], [en línea] disponible en: http://www.unal.edu.co/contenido/sobre un/sobreun vision.htm .

6. Hidrovo A. Documento técnico: Sistema de Vigilancia Ocupacional de Trastornos Osteomusculares (SIVIGO-TOM). Bogotá. 2001; 7-22.

7.Instituto Nacional para la Seguridad y Salud Ocupacional NIOSH. Publicación 95119. 1997, [citado en 26 de noviembre de 2009], [en línea] disponible en: http://www.cdc.gov/spanish/niosh/fact-sheets/fact-sheet-705005.html .

8.Piedrahita, $\mathrm{H}$. Evidencias epidemiológicas entre factores de riesgo en el trabajo y los desórdenes musculoesqueléticos. MAPFRE Medicina 2004; 15 (3): 212-221.

9.Ministerio de Salud, República de Colombia. Resolución № 008430 de 1993 (4 de octubre de 1993), Santa fe de Bogotá. 1993; 1-12.

10. Consejo de Organizaciones Internacionales de las Ciencias Médicas. Pautas éticas internacionales para la investigación biomédica en seres humanos preparadas por el consejo de organizaciones internacionales de las ciencias médicas (CIOMS) en colaboración con la Organización Mundial de la Salud, Chile. 2003; 21-87.

11. Velandia E. Enfermedad Profesional y su Impacto Social. Consejo Colombiano de Seguridad. FASECOLDA. Junio de 2008.

12. García M y Castañeda R. Enfermedades Profesionales declaradas en hombres y mujeres en España en 2004. Revista Española Salud Pública 2006; 80 (4): 361-375.

13. Rohlfs I, et al. La importancia de la perspectiva de género en las encuestas de salud. Gaceta Sanitaria. 2000; 14 (2): 146-155.

14. Pålsson B, Strömberg U, Ohlsson K, Skerfving S. Absence attributed to incapacity and occupational disease/accidents among female and male workers in the fishprocessing industry. Occup Med (Lond). 1998; 48 (5): 289-295, [citado en 22 de noviembre de 2009], [en línea] disponible en: http://occmed.oxfordjournals.org/cgi/reprint/48/5/289

15. Leijon $\mathrm{O}$, Härenstam A, Waldenström K, Alderling M, Vingård E. Target groups for prevention of neck/shoulder and low back disorders: An exploratory cluster analysis of working and living conditions. Work. 2006; 27 (2): 189-204, [citado en 22 de noviembre de 2009], [en línea] disponible en: http://www.ncbi.nlm.nih.gov/pubmed/16971766?itool=EntrezSystem2.PEntrez.Pubmed .Pubmed_ResultsPanel.Pubmed_RVDocSum\&ordinalpos $=1$

16. Kaergaard A, Andersen JH. Musculoskeletal disorders of the neck and shoulders in female sewing machine operators: prevalence, incidence, and prognosis. Occup Environ Med. 2000; 57 (8): 528-534, [citado en 22 de noviembre de 2009], [en línea] disponible

en:

http://www.ncbi.nlm.nih.gov/pmc/articles/PMC1740005/pdf/v057p00528.pdf

17. Shahla M Eltayeb, J Bart Staal, Amar A Hassan, Salwa S Awad, and Rob A de Bie. Complaints of the arm, neck and shoulder among computer office workers in Sudan: a prevalence study with validation of an Arabic risk factors questionnaire. BMC Environ Health. 2008; 7 (33): 1-11, [citado en 22 de noviembre de 2009], [en línea] disponible en: http://www.ncbi.nlm.nih.gov/pmc/articles/PMC2474607/pdf/1476-069X7-33.pd

18. Melchior M, et. al. Why are manual workers at high risk of upper limb disorders? The role of physical work factors in a random sample of workers in France (the Pays 
de la Loire study). Occup Environ Med. 2006; 63 (11): 754-761, [citado en 22 de noviembre de 2009], [en línea] disponible en: http://www.ncbi.nlm.nih.gov/pmc/articles/PMC2077990/pdf/754.pdf

19. Hagen KB, Magnus P, Vetlesen K. Neck/shoulder and low-back disorders in the forestry industry: relationship to work tasks and perceived psychosocial job stress. Ergonomics. 1998; 41 (10): 1510-1518, [citado en 22 de noviembre de 2009], [en línea] disponible

en: http://www.informaworld.com/smpp/ftinterface $\sim$ content=a713808014 fulltext=7132409 30

20. Punnett L, Gold J, Katz J, Gore R, and Wegman D. Ergonomic stressors and upper extremity musculoskeletal disorders in automobile manufacturing: a one year follow up study. Occup Environ Med. 2004; 61 (8): 668-674, [citado en 22 de noviembre de 2009], [en línea] disponible en: http://www.ncbi.nlm.nih.gov/pmc/articles/PMC1740826/pdf/v061p00668.pdf

21. Malchaire JB, Roquelaure $\mathrm{Y}$, Cock N, Piette A, Vergracht S, Chiron H. Musculoskeletal complaints, functional capacity, personality and psychosocial factors. Int Arch Occup Environ Health. 2001; 74 (8): 549-557, [citado en 22 de noviembre de 2009], [en línea] disponible https://commerce.metapress.com/content/cwtg54jeng609y66/resource-

secured/?target=fulltext.pdf\&sid=toyipmf12jm0osuktucamu $45 \&$ sh=www.springerlink.co $\underline{\mathrm{m}}$

21. Lee HY, Yeh WY, Chen CW, Wang JD. Prevalence and psicosocial risk factors of upper extremity musculoskeletal pain in industries of Taiwan: A Nationwide Study. J Occup Health. 2005; 47 (4): 311-8, [citado en 22 de noviembre de 2009], [en línea] disponible en: http://www.jstage.jst.go.jp/article/joh/47/4/311/_pdf

22. Sim J, Lacey $R$ and Lewis $M$. The impact of workplace risk factors on the occurrence of neck and upper limb pain: a general population study. BMC Public Health. 2006; 6 (234): 1-10, [citado en 22 de noviembre de 2009], [en línea] disponible en: http://www.biomedcentral.com/content/pdf/1471-2458-6-234.pdf

23. European agency for safety and health at work. OSH in Figures: Work-related Musculoskeletal Disorders in the EU. Facts and Figures. En: European Risk Observatory Report. Luxemburgo. 2010. 176 p.

24. Organización Internacional del Trabajo. Technical Backgrounder on the Problematic Diseases in the Proposed List to Replace the List Annexed to the List of Occupational Diseases Recommendation. MERLOD. 2002; 200 (194): 5.

25. Wasiak R y Pransky G. The Impact of Procedure Type, Jurisdiction and Other Factors in Workers. Compensation on Work-Disability Outcomes Following Carpal Tunnel Surgery. Work. 2007; 28: 103-110.

26. Nieuwenhuyse AV, et al. Physical characteristics of the back are not predictive of low back pain in healthy workers: A prospective study. BMC Musculoskeletal Disorders 2009, 10 (2):1-9, [citado en 22 de noviembre de 2009], [en línea] disponible en: http://www.biomedcentral.com/1471-2474/10/2

27. Ministerio de Protección Social. Primera Encuesta Nacional de Condiciones de Salud y Trabajo en el Sistema General de Riesgos Profesionales (I ENCST). Bogotá Colombia. 2007

28. Torada R y Moreno N. Salud Laboral y Género. Capitulo publicado en Mujer y Trabajo. Problemática Actual. Valencia. Editorial Germania, [citado en 2 de noviembre de 2011], [en línea] disponible en: http://www.istas.net/upload/salu\%20laboral\%20y\%20g\%C3\%A9nero.doc . 
ISSN 1695-6141

(๖) COPYRIGHT Servicio de Publicaciones - Universidad de Murcia 